Article

\title{
Impacts of COVID-19 on Electric Vehicle Charging Behavior: Data Analytics, Visualization, and Clustering
}

\author{
Sakib Shahriar (D) and A. R. Al-Ali *(D) \\ Department of Computer Science and Engineering, American University of Sharjah, Sharjah P.O. Box 26666, \\ United Arab Emirates; b00058710@aus.edu \\ * Correspondence: aali@aus.edu
}

check for updates

Citation: Shahriar, S.; Al-Ali, A.R Impacts of COVID-19 on Electric Vehicle Charging Behavior: Data

Analytics, Visualization, and

Clustering. Appl. Syst. Innov. 2022, 5, 12. https://doi.org/10.3390/ asi5010012

Received: 21 November 2021

Accepted: 4 January 2022

Published: 7 January 2022

Publisher's Note: MDPI stays neutral with regard to jurisdictional claims in published maps and institutional affiliations.

Copyright: (C) 2022 by the authors. Licensee MDPI, Basel, Switzerland. This article is an open access article distributed under the terms and conditions of the Creative Commons Attribution (CC BY) license (https:// creativecommons.org/licenses/by/ $4.0 /)$

\begin{abstract}
COVID-19 pandemic has infected millions and led to a catastrophic loss of lives globally. It has also significantly disrupted the movement of people, businesses, and industries. Additionally, electric vehicle (EV) users have faced challenges in charging their vehicles in public charging locations where there is a risk of COVID-19 exposure. However, a case study of EV charging behavior and its impacts during the SARS-CoV-2 is not addressed in the existing literature. This paper investigates the impacts of COVID-19 on EV charging behavior by analyzing the charging activity during the pandemic using a dataset from a public charging facility in the USA. Data visualization of charging behavior alongside significant timelines of the pandemic was utilized for analysis. Moreover, a cluster analysis using k-means, hierarchical clustering, and Gaussian mixture models was performed to identify common groups of charging behavior based on the vehicle arrival and departure times. Although the number of vehicles using the charging station was reduced significantly due to lockdown restrictions, the charging activity started to pick up again since May 2021 due to an increase in vaccination and easing of public restrictions. However, the charging activity currently still remains around half of the activity pre-pandemic. A noticeable decline in charging session length and an increase in energy consumption can be observed as well. Clustering algorithms identified three groups of charging behavior during the pandemic and their analysis and performance comparison using internal validation measures were also presented.
\end{abstract}

Keywords: low emission vehicles; COVID-19; cluster analysis; charging infrastructure; data analytics; mobility

\section{Introduction}

COVID-19, first detected in Wuhan, Hubei province in China, was officially declared a pandemic by World Health Organization (WHO) on 11 March 2020 [1]. Lockdown restrictions around the globe and social distancing protocols were introduced to minimize the spread. As of 17 August 2021, COVID-19 led to over 200 million infections and 4.4 million deaths [2]. The introduction of vaccines has provided a potential pathway to end the pandemic. However, the emergence of new variants has posed a different challenge toward ending the pandemic [3]. More than 4.7 billion vaccine doses have been administered by 17 August 2021 [2]. Nevertheless, the impacts of COVID-19 were severe on various sectors and industries. This includes the negative impacts on the economy [4], mental health [5], education, and tourism [6]. The pandemic also had an impact on the energy sector. Although the overall electricity consumption was lower due to the lockdown measures, there was an increase in household electricity consumption [7]. The energy sector has become a point of focus due to the emerging climate change concerns.

According to the Intergovernmental Panel on Climate Change (IPCC), impacts of climate change are evident and, in some cases, irreversible [8]. Energy consumption from the transportation sector is significant, accounting for over $25 \%$ of world energy consumption [9]. To provide a more sustainable alternative, research, development, and adoption 
of electric vehicles (EVs) are significantly increasing. Global sales of EVs increased by about $140 \%$ in the first quarter of 2021 compared to the same period in 2020 [10]. This significant growth amidst the pandemic is a promising sign for global EVs transition. EVs on average require longer charging times compared to the refueling of traditional vehicles. This has generally caused skepticism in the adoption of EVs. Fortunately, the improvement in battery technology including faster charging methods and the development of charging infrastructures around the globe [11] are potential solutions to EV charging. The emergence of novel EV technologies including digital twin with connected Internet of Things and bidirectional vehicle-to-grid features [12] promises further growth. However, it is still important to understand the charging behavior in public charging spaces for effective management of charging demand. According to the sustainable development scenario which considers the climate goals, 230 million EVs are projected by 2030 representing $12 \%$ of road vehicles [10]. This massive increase could potentially lead to uncontrolled charging as a result of overwhelming public charging demands in spaces such as shopping malls and universities. Uncontrolled charging could lead to catastrophic impacts on the power grid including overloading of the power equipment and an increase in power losses [13]. Moreover, the availability of charging infrastructure plays a primary role in EV market demand [14] and the mobility behavior of different EV fleets including logistics and office differs greatly in terms of plug-in duration and energy consumption [15]. Therefore, understanding and quantifying public EV charging behavior is essential with the growing charging demands. Data-driven approaches to modeling of charging behavior present a more realistic and comprehensive representation than compared to simulation or mathematical modeling that contains assumptions [16]. However, uncertain scenarios such as a global pandemic impact the accuracy of any modeling. During these times, it is possible that the charging load is unevenly distributed. For example, due to a large number of people staying indoors for a longer period of time, it is likely that more people would utilize residential charging. In addition to the potential overuse of household appliances, this could lead to serious pressure on the electricity grid. As such, these scenarios should be independently studied and integrated into various modeling. Decentralized load frequency controls [17] and fault-tolerant mechanisms [18] could be utilized in these situations. Although existing works have characterized EV charging behavior in various public locations, the focus of this research is on the analysis of charging behavior during the COVID-19 pandemic. Therefore, this research aims to analyze and quantify public charging behavior during lockdown restrictions which have not been addressed by the existing body of literature.

The proposed research work has several implications. First, the analysis presented can be utilized by researchers, the public energy sector, and policymakers to make better decisions during a future lockdown or similar public restrictive scenarios. This would enable the energy sector to adjust the time of use electricity price to match the changing demand without compromising on the user's flexibility. Moreover, the key insights identified, such as the increase in energy consumption despite a decrease in charging activity due to the lockdowns, can help public charging providers to better manage their electricity demand. This would perhaps lead to incentivizing users to charge their cars at home or locally over a period of time to distribute the increasing energy demands. Finally, the clustering results provide a high-level understanding of the different groups of charging behaviors during the lockdown period. The clustering results indicate that despite the lockdown, users in the same group follow a routine in their daily charging activities. Following are the key contributions of this paper:

It analyzes the charging data from a public charging facility in the state of California, USA during the COVID-19 pandemic. To the best of our knowledge, this is the first research addressing the pandemic EV charging behavior.

It utilizes K-means, hierarchical, and Gaussian mixture models to find clusters of charging behavior during the pandemic. 
It analyses and quantifies the clustering results and provides a discussion on the future work.

The rest of the paper is organized as follows: Section 2 presents a concise review of the existing works in the literature as well as a description of clustering algorithms. The dataset description, preprocessing, and in-depth analysis of charging behavior during COVID-19 are presented in Section 3. In Section 4, the clustering results are presented and discussed. Section 5 concludes the paper and outlines the future research directions.

\section{Background}

\subsection{Related Works}

Existing research works have utilized data-driven approaches and clustering solutions for characterizing EV charging behavior. Shen et al. [19] utilized the K-means algorithm to find clusters of charging behavior. Three clusters were obtained using features that included connection time and departure time. Based on simulation results, two of the three cluster groups were identified as stable and predictable users whereas the third cluster was identified as unpredictable users. K-nearest neighbor (K-NN) algorithm was then used to classify these two groups. Similarly, four clusters of charging behavior were identified using hierarchical clustering in [20]. Most of the users belonged to the first cluster, where the common charging behavior was to charge during the night and in the morning. The users in the second cluster preferred to charge after work during the evening. The third cluster users charged throughout the day and the fourth cluster users charged in the late evening. Helmus et al. [21] discovered thirteen clusters of behavior using the Gaussian mixture model. Seven of these clusters contained daytime charging sessions with varying lengths of charging, and six of them contained overnight charging sessions. They also utilized k-medoids clustering to find nine distinct clusters. Among these, three clusters contained daytime chargers, three contained overnight chargers, and three contained nontypical groups of chargers. In [22], the authors proposed a machine learning approach to learn charging behavior by considering different time granularity. Two independent models were first developed to predict the registered users and unregistered users, respectively. The results were then combined to make the final predictions. Extreme gradient boosting (XGBoost) performed the best and the prediction accuracy was the highest with a 15-min granularity. Similarly, [23] utilized a machine learning approach to predict charging behavior with added weather, traffic, and events information to the historical charging data. They reported the best performance with an ensemble model which outperformed existing approaches. Barthel et al. [15] utilized real-world charging data from Germany to explain temporal and power-specific flexibility characteristics of three vehicle fleets namely pool vehicles of office employees, a public authority, and a logistics company. Their analysis revealed a variability in charging behavior among the fleets with a higher charging flexibility in the logistics group.

From the related works in the literature, only one research work has focused on EV impacts during COVID-19. Wen et al. [24] studied the impacts of COVID-19 on the Chinese EVs industry. Various economic trends in EV sales were discovered including a short-term reduction of sales during COVID-19. They also identified an interruption of materials import due to travel restrictions leading firms to domestic production of critical EV parts. Although previous works in the literature have addressed EV charging behavior, they are based on pre-pandemic conditions. Therefore, to better understand user charging comportment during COVID-19 and pandemics or lockdown scenarios in general, it is essential to analyze and model the data during the pandemic period.

\subsection{Clustering Algorithms and Evaluation}

To find clusters of common charging behavior, three popular clustering algorithms were utilized, namely k-means, hierarchical, and gaussian mixture model (GMM). These clustering algorithms are widely used in clustering applications related to charging behavior 
in the existing literature [16]. Furthermore, for the limited dataset size, more complex deep learning-based clustering solutions were not appropriate.

In k-means clustering, the data points are initially assigned randomly to k-centroid points, as described in Algorithm 1. $K$ is defined as the number of clusters. The elbow method is usually utilized to define the number of clusters [25]. The data points are then iteratively assigned to new centroids based on their similarity using Equation (1). Meanwhile, the centroids are computed and every iteration is updated based on Equation (2). The process is repeated until the algorithm converges and the cluster labels do not change any further.

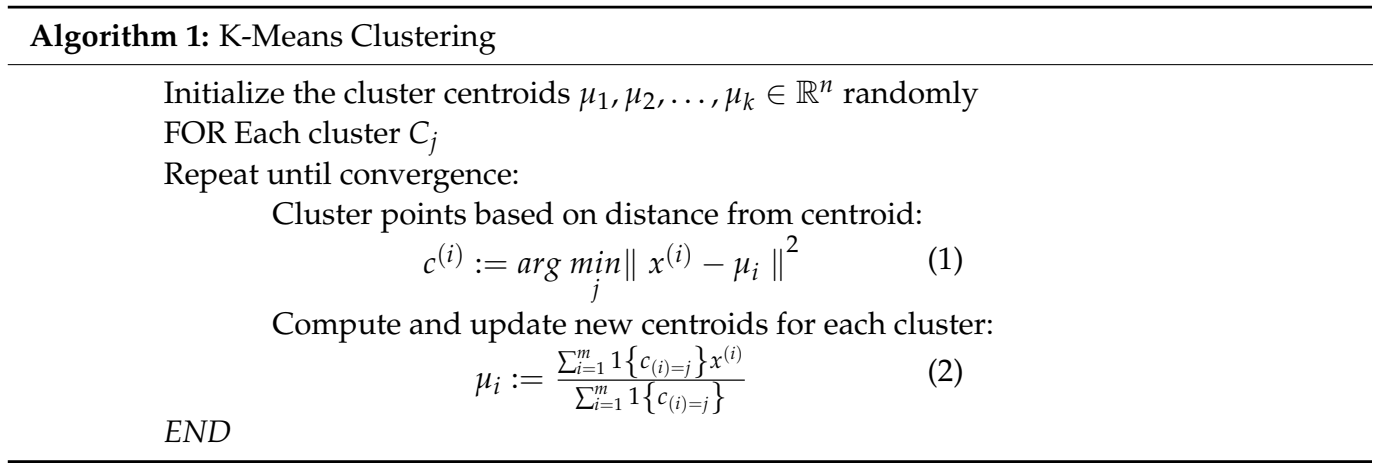

Hierarchical clustering used in the agglomerative variation results in the clustering occurring in a bottom-up approach. Each item starts off as an individual cluster and is then merged as one moves up the hierarchy [26]. Gaussian mixture model was also used to cluster the common charging behavior. In this approach, probabilistic learning takes place that can represent normally distributed subpopulations by considering multiple normal distributions of the dataset [16].

Internal validation was utilized to quantify the performance of clustering since no ground truth was available. Three internal validation metrics namely Silhouette index, Davies-Bouldin index, and Calinski-Harabasz index were used. For a given cluster, $X_{j}(j=1, \ldots c)$, the silhouette index is defined in Equation (3) [27]:

$$
s(i)=\frac{(y(i)-z(i))}{\operatorname{Max}\{y(i), z(i)\}}
$$

where $y(i)$ is the average distance between the $i$ th sample and all of the samples included in $X_{j}$, and $z(i)$ is the minimum average distance between the $i$ th sample and all of the samples clustered in $X_{k}(k=1, \ldots c ; k \neq j)$. The objective in the Davies-Bouldin index, defined in Equation (4), is to identify sets of clusters that are closely packed but separated well from other clusters [27].

$$
\text { Davies - Bouldin }=\frac{1}{c} \sum_{i=1}^{c} \operatorname{Max}_{i \neq j}\left\{\frac{z\left(X_{i}\right)+z\left(X_{j}\right)}{d\left(c_{i}, c_{j}\right)}\right\}
$$

where $c$ denotes the number of clusters, $i, j$ are cluster labels. Samples in clusters $i$ and $j$ to their respective cluster centroids are defined by $z\left(X_{i}\right) z\left(X_{j}\right)$ and $d\left(c_{i}, c_{j}\right)$ is the distance between the centroids. Finally, the Calinski-Harabasz index is defined by Equation (5):

$$
\text { Calinski }- \text { Harabasz }=\frac{\operatorname{trace}\left(S_{B}\right)}{\operatorname{trace}\left(S_{I}\right)} \cdot \frac{n_{S}-1}{n_{S}-c}
$$

where $c$ denotes the number of clusters, $n_{S}$ denotes the number of clustered samples, $S_{B}$ defines the between-cluster scatter matrix, and $S_{I}$ defines the internal scatter matrix. 


\section{Impacts of COVID-19 on EVs Charging}

\subsection{Dataset and Preprocessing}

The adaptive charging network ( $\mathrm{ACN}$ ) dataset containing more than 30,000 charging sessions [28] was used for this study. The dataset contains charging records from two charging spaces in Caltech University campus, California. The Jet Propulsion Laboratory (JPL) charging facility is only accessible to employees and therefore, only the data from Caltech, which is open to the public, was used. The dataset contains logged charging events as well as user-related attributes. The charging events include the arrival time, departure time, and energy consumption. Meanwhile, the user-related data consist of users' energy requests and their estimated departure times. For this study, data containing the charging events from 1 February 2020, until the end of July 2021 were considered. This enables the analysis of trends in charging behavior throughout the pandemic. Since user-related fields were not used in this study, the dataset did not contain any missing values. Overall, a total of 3787 records were obtained from the Caltech site for the specified period of time. The preprocessing steps involved converting the arrival and departure time values to a suitable numeric format. This was achieved by dividing the minute by 60 and adding to the hour, such that 10:17 was converted to 10:28, for example. Moreover, using the datetime method from the pandas [29] library in python, the length of charging sessions was obtained by subtracting the arrival time from the departure time.

\subsection{Data Analysis}

In terms of graphical analysis, the objective was to understand the ways in which the charging behavior evolved throughout the pandemic. More specifically, the goal was to find out the impacts on the charging activity and the overall charging behavior.

The charging activity in terms of the number of charging sessions is presented in Figure 1. The figure is also annotated with the major COVID-19 events in the USA using data from the American Journal of Managed Care [30] and other credible news sources. A high charging activity can be observed at the beginning of February 2020, with about 210 charging sessions per week on average. This is followed by a dramatic decrease in charging activity as the USA declared a public emergency on 3 February and WHO officially declared COVID-19 a pandemic on 11 March. The charging activity continued to decline further as California, where the ACN charging facility is located, became the first state to announce lockdown measures. There was no charging activity between 3 August and 15 November 2020, as the pandemic worsened nationwide. Moreover, the state of California faced a record number of cases and deaths during this period as well as rolling electricity blackouts and wildfires [31], explaining the inactivity. This period also saw a nationwide record of 100,000 cases per day. The charging activity resumed with the promising signs of vaccine developments during November 2020. During December 2020, the activity per week on average was about 50 sessions, as Pfizer and AstraZeneca vaccines received emergency use authorization (EUA). During January and March 2021, there was a slight decline due to increased deaths and reports of new variants. However, with the vaccination rates increasing and the ease of restrictions, the charging activity picked up between May and July 2021. However, the charging activity continues to be lower than pre-pandemic with the current activity of about 110 sessions per week being almost half of the activity just before the pandemic.

To get a better reflection of how the overall EV charging behavior evolved throughout the pandemic, the trend in average arrival and departure time is plotted in Figure 2. Furthermore, the average length of the charging session, i.e., the difference between the arrival and departure time was plotted. It can be noticed that on average most of the charging sessions began during the afternoon, between 12 p.m. and 2 p.m. The sessions usually last for about $3 \mathrm{~h}$ and hence most of the departure time is between 3 p.m. and 5 p.m. However, looking at the session length trend, it is evident that as the pandemic began and progressed through 2020 and 2021, the charging length on average became shorter. This indicates that people spent less time outdoors charging their EVs. This 
trend is reestablished with the monthly average charging length diagram in Figure 3 . The charging length was almost 6 h during February and March 2020, when the pandemic was officially declared. It then dropped to about $4 \mathrm{~h}$ on average between April and August 2020. There was no charging activity in September and October, as indicated in Figure 1. After the resumption of charging activities in November 2020, the average length continued to remain below $3 \mathrm{~h}$ until June 2021. However, in July the average length increased to $3.4 \mathrm{~h}$ indicating a shift toward pre-pandemic charging lengths.

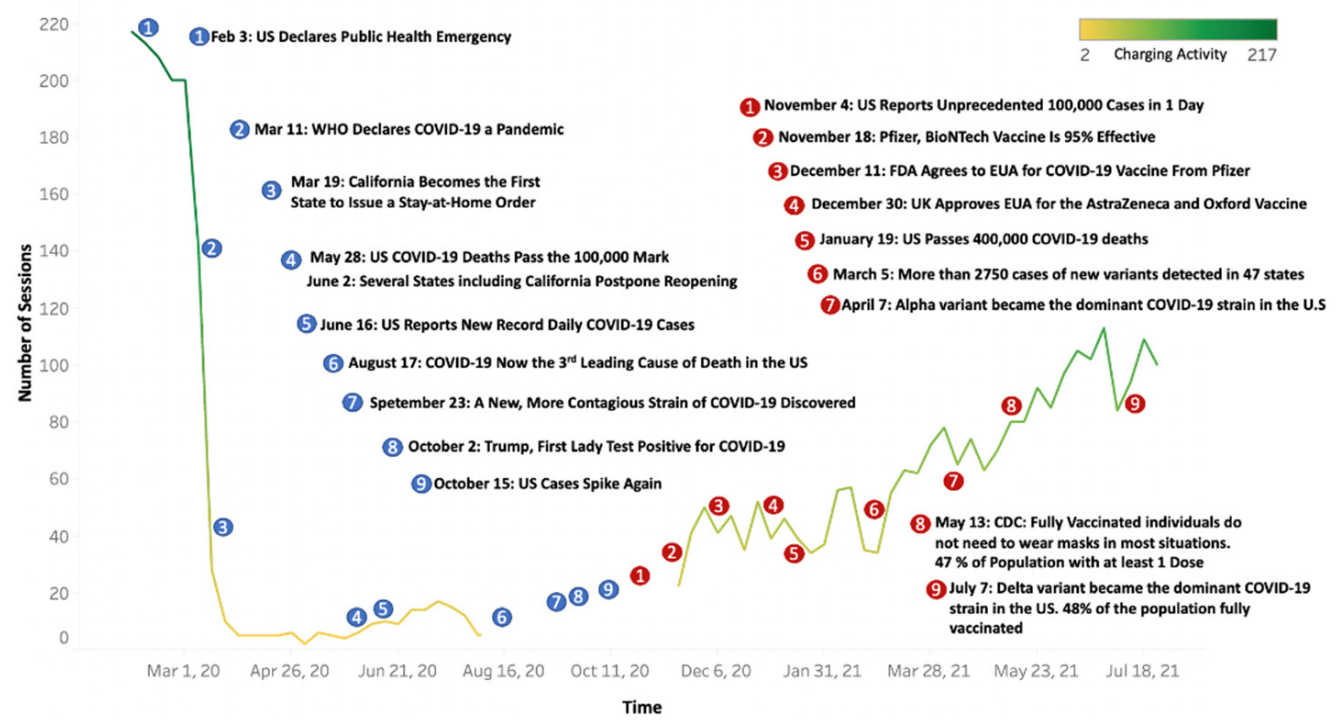

Figure 1. Charging activity by month during COVID-19.

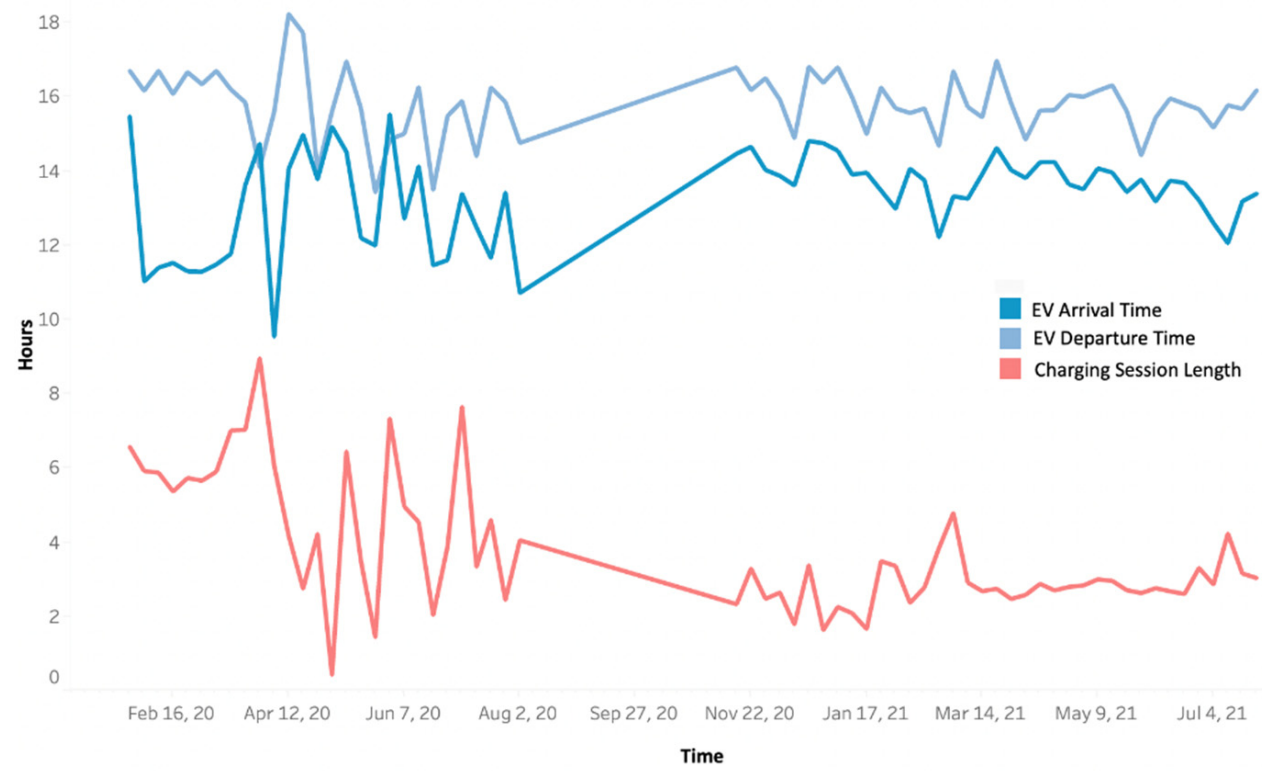

Figure 2. Charging behavior trends during COVID-19.

The decrease in charging length is conflicted with the increase in overall energy consumption, as displayed in Figure 4. The average energy consumption was about $7.4 \mathrm{kWh}$ during February and March 2020. It then increased to $11.8 \mathrm{kWh}$ between April and June 2020. The abnormally high average during August 2020 can be explained by only a total of 5 charging sessions before August 3. After this point, there was no charging activity until 15 November 2020. Therefore, the consumption during August can be ignored for this analysis. From December 2020 until June 2021, the energy consumption continued to 
increase and reached about $13 \mathrm{kWh}$ on average as the charging lengths became shorter. However, as the charging length started to increase again from July 2021, a reduction in energy consumption can be observed. The contrast in the two variables can be explained by the fact that during shorter sessions, more energy is distributed at an increasing rate. It is also likely that many vehicles utilizing the charging facility during this time also exploited fast charging. This is because owners with slower charging vehicles would most likely prefer to charge residentially due to concerns over staying outdoors for a longer period of time.

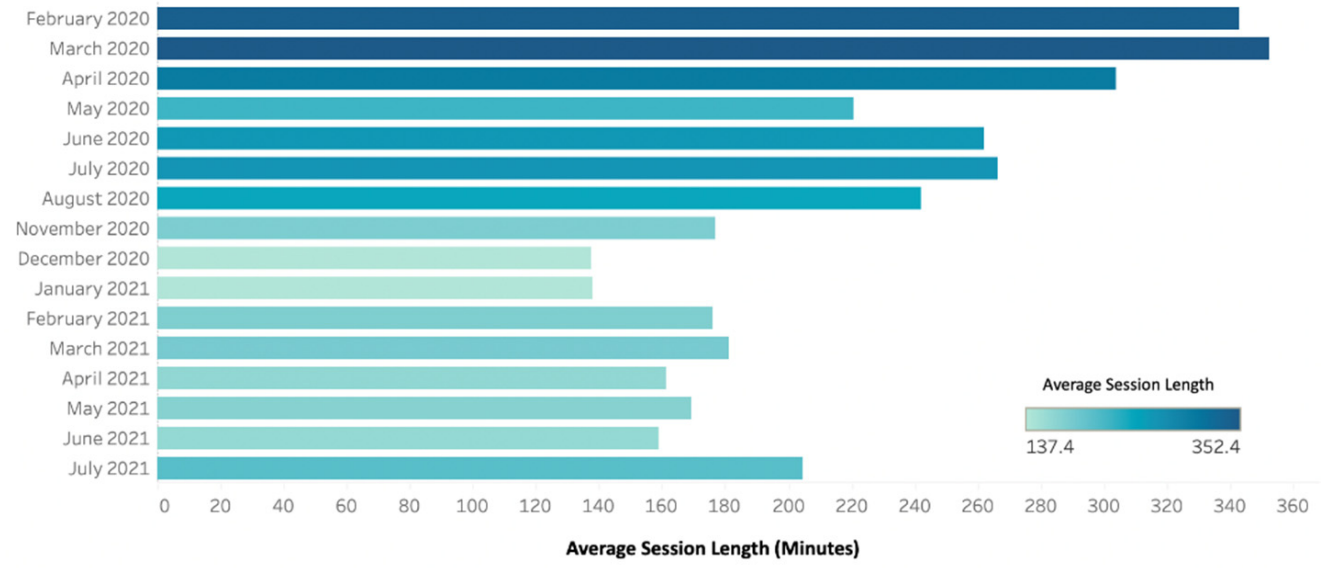

Figure 3. Average charging session length by month during COVID-19.

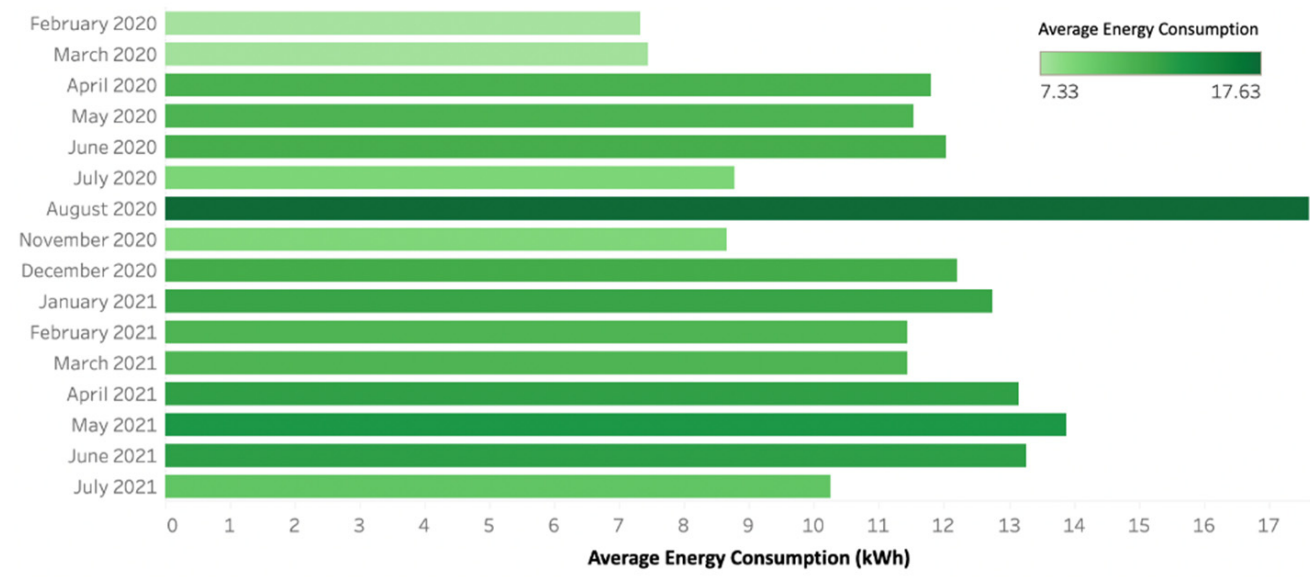

Figure 4. Average energy consumption by month during COVID-19.

\section{Clustering of Charging Behavior during COVID-19}

\subsection{Experimental Setup}

As mentioned in Section 2, the number of clusters was required to be determined before training. Figure 5 illustrates this method, where three clusters were decided based on the sum of squared error (SSE). For all three algorithms, the number of clusters to be found was set to three. Next, all charging sessions beginning March 2020 were selected to find clusters of common behavior. To perform the clustering, the arrival and departure time was selected to identify the charging behaviors. The clustering was implemented in python using scikit-learn [32] library. 


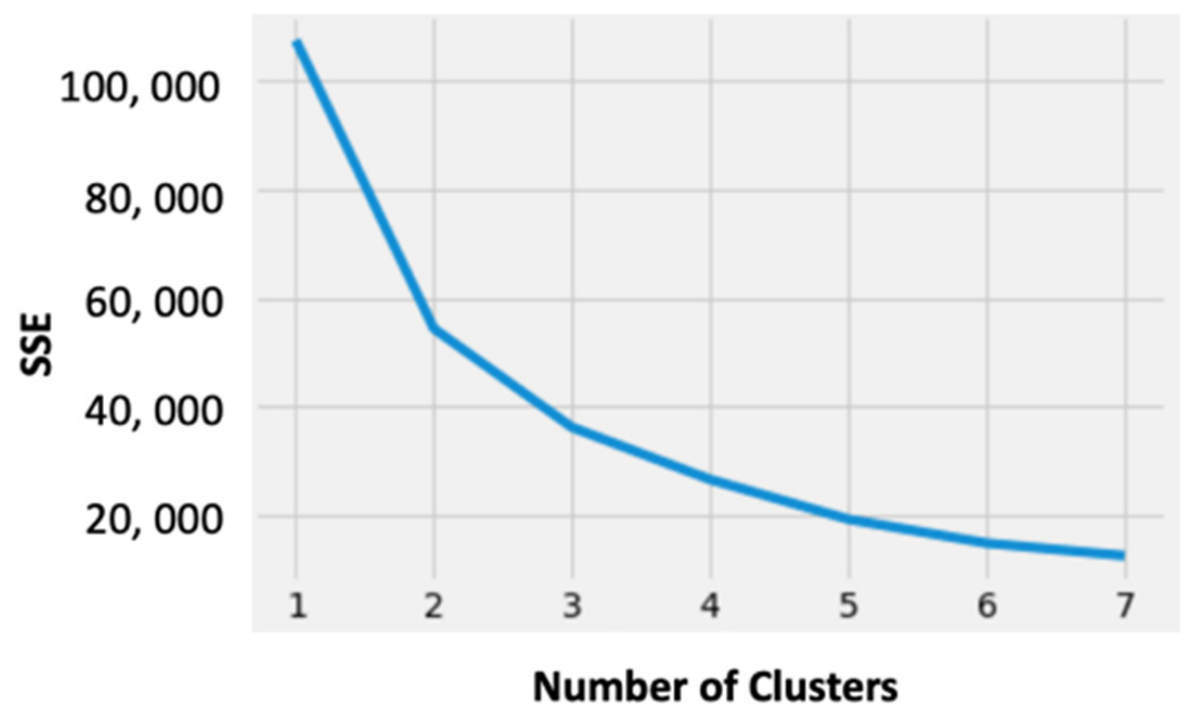

Figure 5. Elbow method for detecting the number of clusters.

\subsection{Clustering Results}

As mentioned earlier, three clusters were selected using the elbow method. Figure 6 presents the clusters obtained using k-means. The three clusters are almost equal in density and well separated. Cluster one in purple represents the early chargers who usually start between 5 a.m. and 10 a.m. and finish charging between 9 a.m. and 3 p.m. However, it also includes a few users who arrive later during the day and finish charging the next morning. The second cluster in red represents the group that arrives in the afternoon between 12 p.m. and 4 p.m. and leaves between 3 p.m. and 8 p.m. Finally, cluster three in green contains users who arrive after 3 p.m. and finish charging before midnight.

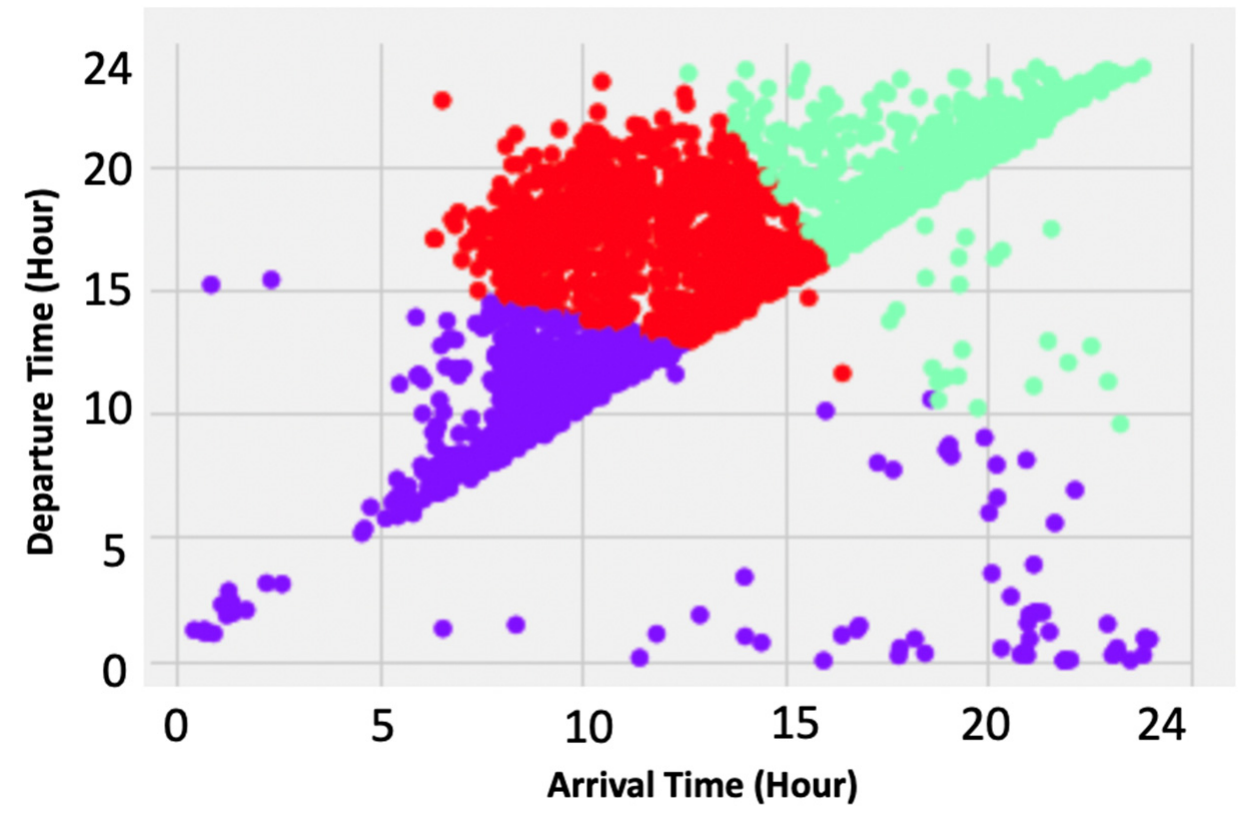

Figure 6. Cluster visualization for K-means.

In Figure 7, the three clusters using agglomerative clustering are presented. The results are similar to k-means clustering in terms of the charging behavior of the three groups. However, cluster two in red is less dense using this algorithm, and therefore, there is a greater overlap between the afternoon chargers, and they belong to all three clusters. 


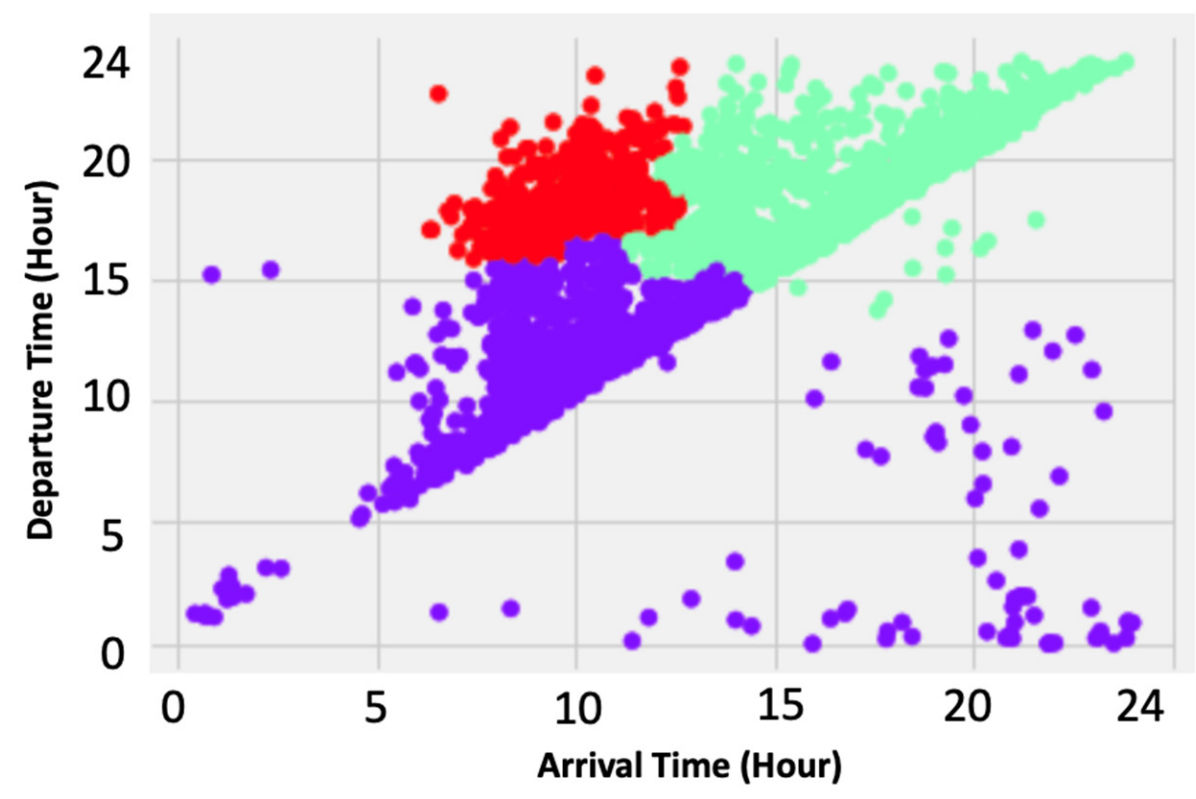

Figure 7. Cluster visualization for hierarchical agglomerative.

Finally, the three clusters using GMM are presented in Figure 8. The three clusters using this algorithm are very different compared to the aforementioned approaches. In this case, cluster one in purple contains most of the sessions where the arrival time is between 7 a.m. and 8 p.m. and the departure time is between 10 a.m. and midnight. The second cluster in red contains shorter sessions where the charging session lasts for about $2 \mathrm{~h}$. The third and smallest cluster in green contains sessions scattered throughout the day.

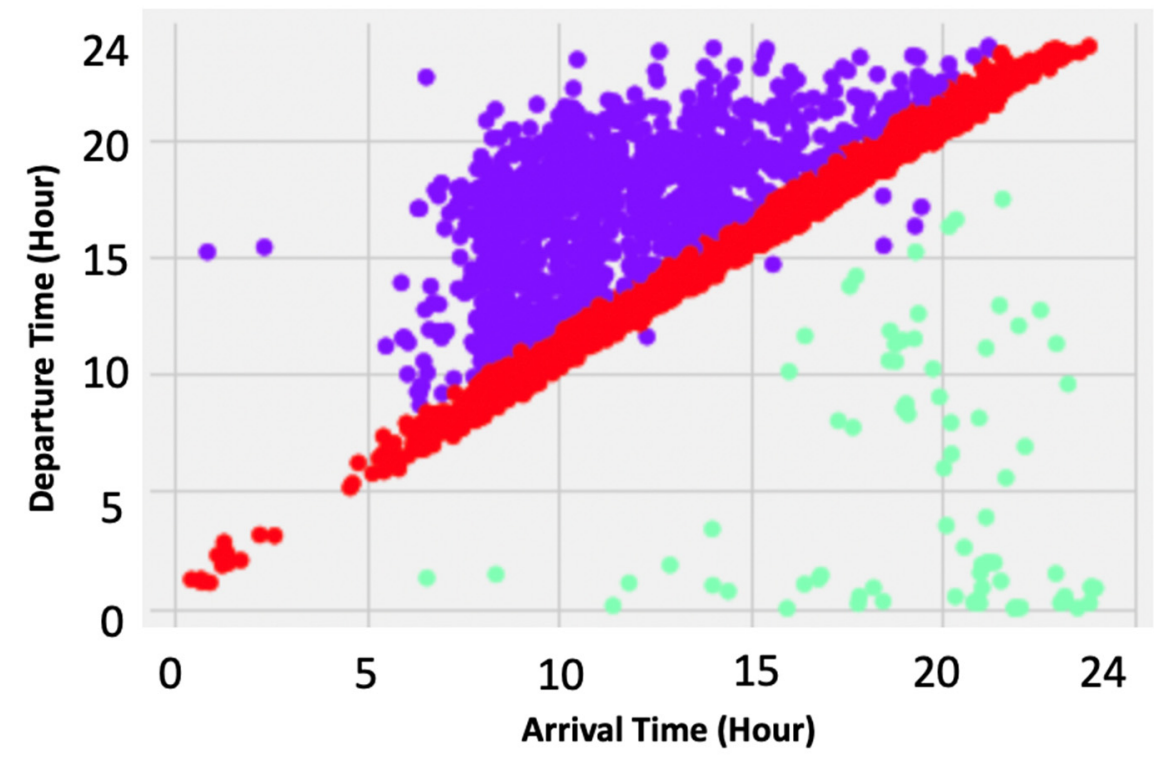

Figure 8. Cluster visualization for GMM.

Table 1 summarizes the results using the three internal validation metrics. Silhouette index is between -1 and +1 and higher scores indicate better clustering. Similarly, a higher value for the Calinski-Harabasz index indicates good clustering. Conversely, the lowest possible score for the Davies-Bouldin index is 0 , and a lower score represents better clustering. The scores in Table 1 indicate that the Gaussian mixture model produced the worst clustering. This is consistent with the cluster diagram in Figure 8. K-means performed the best in terms of Silhouette and Calinski-Harabasz indices, whereas hierarchical clustering performed the best in terms of the Davies-Bouldin index. 
Table 1. Internal validation scores.

\begin{tabular}{cccc}
\hline Clustering/Metrics & Silhouette & Davies-Bouldin & Calinski-Harabasz \\
\hline K-means & 0.41 & 0.85 & 2904 \\
Hierarchical & 0.38 & 0.74 & 2270 \\
GMM & 0.15 & 2.05 & 344 \\
\hline
\end{tabular}

Overall, the results obtained in this work are consistent with the previous work in [19], where three clusters of charging behaviors were identified. However, the authors did not quantify their clustering results. The authors in [20] found four clusters using hierarchical clustering. They also did not present an evaluation of their clustering results. In our work, the quantified results are presented in Table 1 . There are a few limitations in the clustering results in the proposed work. First, the number of clusters determined using the elbow method was used for all three algorithms. It is possible to experiment with more methods to determine the optimal number of clusters. Finally, the charging sessions were clustered based on the arrival and departure times. It is likely that using other parameters such as vehicle type and energy consumption could potentially lead to the discovery of more interesting clusters of behaviors.

\section{Conclusions and Future Work}

This paper presented an analysis of public EV charging behavior during the COVID-19 pandemic. Analysis indicates that the lockdown measures had an impact on the charging activity during the pandemic. It was found that there were no charging sessions between the beginning of August and until the middle of November 2020. Despite the recent growth in charging activity due to increased testing, vaccination, and reduction in lockdown restrictions, the charging activity is currently about half of pre-pandemic. Moreover, the charging behavior evolved with shorter and high-energy sessions during the pandemic. Furthermore, using clustering algorithms, three distinct groups of charging behaviors were identified. The best clustering was obtained using K-means followed by hierarchical clustering. The limitations of the clustering approach include the single method used for determining the number of clusters as well as the reliance on only two parameters for clustering. The proposed work contains several practical implications. The analysis of the public charging behavior during the pandemic and lockdown situations, in general, helps the energy sector and policymakers to better manage the charging demand. Based on the identified trends and patterns, the charging electricity price of use could also be adjusted to control some of the charging load.

For future work, an analysis of residential charging data should be performed. It is likely to show a dramatic increase in charging activities due to the lockdown measures forcing people to charge their vehicles residentially. For optimal decision-making on EV charging demand on a state level, it is essential to combine both residential and nonresidential charging activities over a period of time. Moreover, data from other charging facilities in the USA and across the globe should be used to further compare and validate the findings in this work. Although beneficial, the proposed research is limited by the data source which only characterizes a single location. Given that the pandemic is still ongoing with new variants being identified, a forecast for the year 2022 based on the current data is required. The forecasting model should incorporate statistical, time series, and machine learning approaches. Finally, deep learning and neural approaches to clustering can be experimented with to provide a further comparison. Deep learning-based methods are known to usually provide more accurate solutions with larger datasets and therefore must be explored in future research.

Author Contributions: Conceptualization, S.S. and A.R.A.-A.; Data curation, S.S.; Methodology, S.S. and A.R.A.-A.; Supervision, A.R.A.-A.; Writing-original draft, S.S.; Writing-review \& editing, A.R.A.-A. All authors have read and agreed to the published version of the manuscript. 
Funding: This work was supported in part by the Open Access Program from the American University of Sharjah under Grant OAP-CEN-069.

Institutional Review Board Statement: Not applicable.

Informed Consent Statement: Not applicable.

Data Availability Statement: The dataset can be accessed using: https:/ /ev.caltech.edu/dataset (accessed on 1 June 2020).

Acknowledgments: This paper represents the opinions of the authors and does not mean to represent the position or opinions of the American University of Sharjah.

Conflicts of Interest: The authors declare no conflict of interest.

\section{References}

1. WHO. Archived: WHO Timeline-COVID-19. Available online: https://www.who.int/news/item/27-04-2020-who-timeline--covid-19 (accessed on 16 August 2021).

2. Dong, E.; Du, H.; Gardner, L. An interactive web-based dashboard to track COVID-19 in real time. Lancet Infect. Dis. 2020, 20, 533-534. [CrossRef]

3. Hayawi, K.; Shahriar, S.; Serhani, M.A.; Alashwal, H.; Masud, M.M. Vaccine versus Variants (3Vs): Are the COVID-19 Vaccines Effective against the Variants? A Systematic Review. Vaccines 2021, 9, 1305. [CrossRef]

4. Ozili, P.K.; Arun, T. Spillover of COVID-19: Impact on the Global Economy. 2020. Available online: https://dx.doi.org/10.2139/ ssrn.3562570 (accessed on 16 August 2021).

5. Kumar, A.; Nayar, K.R. COVID 19 and its mental health consequences. J. Ment. Health 2021, 30, 1-2. [CrossRef] [PubMed]

6. Škare, M.; Soriano, D.R.; Porada-Rochoń, M. Impact of COVID-19 on the travel and tourism industry. Technol. Forecast. Soc. Chang. 2021, 163, 120469. [CrossRef]

7. Krarti, M.; Aldubyan, M. Review analysis of COVID-19 impact on electricity demand for residential buildings. Renew. Sustain. Energy Rev. 2021, 143, 110888. [CrossRef]

8. Masson-Delmotte, V.; Zhai, P.; Pirani, A.; Connors, S.L.; Péan, C.; Berger, S.; Caud, N.; Chen, Y.; Goldfarb, L.; Gomis, M.I. (Eds.) Climate Change 2021: The Physical Science Basis. Contribution of Working Group I to the Sixth Assessment Report of the Intergovernmental Panel on Climate Change; Cambridge University Press: Cambridge, UK, 2021.

9. IEA. Key World Energy Statistics 2018-Analysis. Available online: https:/ /www.iea.org/reports/ key-world-energy-statistics2019 (accessed on 1 June 2020).

10. IEA. Global EV Outlook 2021-Analysis. Available online: https://www.iea.org/reports/global-ev-outlook-2021 (accessed on 16 August 2021).

11. Mandrile, F.; Cittanti, D.; Mallemaci, V.; Bojoi, R. Electric vehicle ultra-fast battery chargers: A boost for power system stability? World Electr. Veh. J. 2021, 12, 16. [CrossRef]

12. Van Mierlo, J.; Berecibar, M.; El Baghdadi, M.; De Cauwer, C.; Messagie, M.; Coosemans, T.; Jacobs, V.; Hegazy, O. Beyond the state of the art of electric vehicles: A fact-based paper of the current and prospective electric vehicle technologies. World Electr. Veh. J. 2021, 12, 20. [CrossRef]

13. Clement-Nyns, K.; Haesen, E.; Driesen, J. Analysis of the impact of plug-in hybrid electric vehicles on residential distribution grids by using quadratic and dynamic programming. World Electr. Veh. J. 2009, 3, 214-224. [CrossRef]

14. Wróblewski, P.; Drożdż, W.; Lewicki, W.; Miazek, P. Methodology for assessing the impact of aperiodic phenomena on the energy balance of propulsion engines in vehicle electromobility systems for given areas. Energies 2021, 14, 2314. [CrossRef]

15. Barthel, V.; Schlund, J.; Landes, P.; Brandmeier, V.; Pruckner, M. Analyzing the charging flexibility potential of different electric vehicle fleets using real-world charging data. Energies 2021, 14, 4961. [CrossRef]

16. Shahriar, S.; Al-Ali, A.R.; Osman, A.H.; Dhou, S.; Nijim, M. Machine learning approaches for ev charging behavior: A review. IEEE Access 2020, 8, 168980-168993. [CrossRef]

17. Haesalhelou, H.; Parthasarathy, H.; Nagpal, N.; Agarwal, V.; Nagpal, H.; Siano, P. Decentralised stochastic disturbance observerbased optimal frequency control method for interconnected power systems with high renewable shares. IEEE Trans. Ind. Inform. 2021, 1. [CrossRef]

18. Alhelou, H.A.H.; Cuffe, P. A Dynamic state estimator based tolerance control method against cyberattack and erroneous measured data for power systems. IEEE Trans. Ind. Inform. 2021, 1. [CrossRef]

19. Shen, Y.; Fang, W.; Ye, F.; Kadoch, M. EV charging behavior analysis using hybrid intelligence for 5G smart grid. Electronics 2020, 9, 80. [CrossRef]

20. Gerossier, A.; Girard, R.; Kariniotakis, G. Modeling and forecasting electric vehicle consumption profiles. Energies 2019, $12,1341$. [CrossRef]

21. Helmus, J.R.; Lees, M.H.; Hoed, R.V.D. A data driven typology of electric vehicle user types and charging sessions. Transp. Res. Part C Emerg. Technol. 2020, 115, 102637. [CrossRef] 
22. Qiao, F.; Lin, S. Data-Driven Prediction of Fine-Grained EV Charging Behaviors in Public Charging Stations: Poster. In Proceedings of the Twelfth ACM International Conference on Future Energy Systems, New York, NY, USA, 28 June-2 July 2021; pp. 276-277. [CrossRef]

23. Shahriar, S.; Al-Ali, A.R.; Osman, A.H.; Dhou, S.; Nijim, M. Prediction of EV charging behavior using machine learning. IEEE Access 2021, 9, 111576-111586. [CrossRef]

24. Wen, W.; Yang, S.; Zhou, P.; Gao, S. Impacts of COVID-19 on the electric vehicle industry: Evidence from China. Renew. Sustain. Energy Rev. 2021, 144, 111024. [CrossRef]

25. Kodinariya, T.M.; Makwana, P.R. Review on determining number of Cluster in K-Means Clustering. Int. J. 2013, 1, 90-95.

26. Zualkernan, I.A.; Pasquier, M.; Shahriar, S.; Towheed, M.; Sujith, S. Using BLE beacons and Machine Learning for Personalized Customer Experience in Smart Cafés. In Proceedings of the 2020 International Conference on Electronics, Information, and Communication (ICEIC), Barcelona, Spain, 19-22 January 2020; pp. 1-6. [CrossRef]

27. Rendón, E.; Abundez, I.; Arizmendi, A.; Quiroz, E.M. Internal versus external cluster validation indexes. Int. J. Comput. Commun. 2011, 5, 27-34.

28. Lee, Z.J.; Li, T.; Low, S.H. ACN-Data: Analysis and Applications of an Open EV Charging Dataset. In Proceedings of the Tenth ACM International Conference on Future Energy Systems, New York, NY, USA, 25-28 June 2019; pp. 139-149. [CrossRef]

29. McKinney, W. Pandas: A foundational Python library for data analysis and statistics. Python High Perform. Sci. Comput. 2011, 14, $1-9$.

30. AJMC. A Timeline of COVID-19 Developments in 2020. Available online: https://www.ajmc.com/view/a-timeline-of-covid19developments-in-2020 (accessed on 17 August 2021).

31. Procter, R. Remember When? Timeline Marks Key Events in California's Year-Long Pandemic Grind. CalMatters. 4 March 2021. Available online: https:/ / calmatters.org/health/coronavirus/2021/03/timeline-california-pandemic-year-key-points / (accessed on 17 August 2021).

32. Pedregosa, F.; Varoquaux, G.; Gramfort, A.; Michel, V.; Thirion, B.; Grisel, O.; Blondel, M.; Prettenhofer, P.; Weiss, R.; Dubourg, V.; et al. Scikit-learn: Machine learning in Python. J. Mach. Learn. Res. 2011, 12, 2825-2830. 